\title{
BMJ Open Effects of a new parallel primary healthcare centre and on-campus training programme on history taking, physical examination skills and medical students' preparedness: a prospective comparative study in Taiwan
}

Ying-Ying Yang, ${ }^{1,2,3}$ Shuu-Jiun Wang, ${ }^{1,4}$ Ling-Yu Yang, ${ }^{1,5}$ Jiing-Feng Lirng, ${ }^{1,4}$ Chia-Chang Huang, ${ }^{1,2}$ Jen-Feng Liang, ${ }^{1,5}$ Fa-Yauh Lee, ${ }^{1,3,4}$ Shinn-Jang Hwang, ${ }^{1,4}$ Chin-Chou Huang, ${ }^{1,2}$ Ralph Kirby ${ }^{1}$

To cite: Yang Y-Y, Wang S-J, Yang L-Y, et al. Effects of a new parallel primary healthcare centre and on-campus training programme on history taking, physical examination skills and medical students' preparedness: a prospective comparative study in Taiwan. BMJ Open 2017;7:e016294. doi:10.1136/ bmjopen-2017-016294

- Prepublication history and additional material for this paper are available online. To view please visit the journal online (http://dx.doi.org/10.1136/ bmjopen-2017-016294).

Received 5 February 2017 Revised 18 July 2017 Accepted 3 August 2017

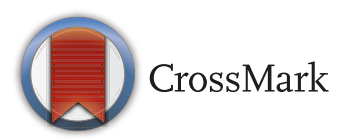

For numbered affiliations see end of article.

Correspondence to Dr Ying-Ying Yang; yangyy@vghtpe.gov.tw

\section{ABSTRACT}

Objectives The primary healthcarecentre (PHCC) is the first place that medical students experience patient contact. Usually, medical students are frustrated by a lack of proper skills training for on-campus history taking (HT), physical examination (PE) and self-directed learning (SDL) to prepare for their PHCC and inhospital patient contact. For pre-clerks, this study aims to compare the effectiveness of PHCC training and PHCC training in combination with on-campus HT and PE training modules (PHCC+on-campus) on their clerkship preparedness.

Design This comparative study utilised prospective, consecutive, end of pre-clerkship group objective structured clinical examination (GOSCE), beginning of clerkship OSCE and self-administered Preparation for Hospital Practice Questionnaire (PHPQ).

Setting/participants 128 pre-clinical clerk volunteers (64 each year) receiving PHCC training (7 week PHCCtraining in addition to 7 week assignment based group learning, academic year 2014, controls) and PHCC training in combination with on-campus module training (academic year 2015,7 week PHCCtraining in addition to 7 week on-campus sessions) were sequentially assessed before the module (week 1), at the end of the module (week 14) and at the beginning of clerkship (week 25).

Results For overall HT and PE skills, both PHCC and PHCC+on-campus module trained pre-clerks performed better on OSCE than GOSCE. Additionally, the improvement was accompanied by higher self-reported PHPQ scores in 'confidence/coping' and 'SDL' domains. At the end of the preclerkship and the beginning of the clerkship stages, the degree of improvement in preparedness in 'confidence/coping' and 'SDL' domains was higher for those in the PHCC+on-campus group than for those in the PHCC group. Among the PHCC+oncampus module participants, a positive association was observed between high mean PHPQ-SDL scores and high OSCE scores.

Conclusions Our study suggests that the PHCC+on-campus module, which is paired faculty led and pre-trained dyad student assisted, is effective in developing a preclinical clerk's

\section{Strengths and limitations of this study}

- Similar preparation period before assessments, as well as randomised raters, simulation patients, students and standardised processes in group objective structured clinical examination (GOSCE)/ OSCE ensured the comparability of the results of the primary healthcare centre (PHCC) group and the PHCC training in combination with on-campus history taking (HT) and physical examination (PE) training (PHCC+on-campus) group.

- Pre-clerks in both modules, which equally extended the whole fourth year of medical education, were volunteers that were more committed, brighter and motivated; this may have resulted in some degree of selective bias.

- This study evaluated the degree of trained skills degradation over time by comparing each participant's performance at the end of the pre-clerk module GOSCE with the beginning of their clerkship OSCE performance.

- Due to the ethics of students' rights of learning, this study was limited by a lack of a true control group, as PHCC module pre-clerks still received $\mathrm{HT}$ and PE skills training.

- The lack of a 'control group,' with pure regular clerkship, limits clarifying whether repetitive practising on simulation patients and receiving structured feedback from well trained faculty in the PHCC+on-campus group will decrease skills degradation over time.

HT and PE skills and intensifying SDL/patient management abilities to prepare for hospital practice in clerkship.

\section{INTRODUCTION}

Taiwan's medical training consists of 7 years of post-secondary medical school followed by 
residency and fellowship training. During pre-clerkship, the fourth year medical students (pre-clerks) receive intensive training in fundamental clinical skills to ensure preparation for clinical clerkship. Then, medical students enter clinical clerkship in their fifth year, followed by internship for primary care of patients. Generally, pre-clerks are not preoccupied with attempts to make diagnoses and perform management beyond their abilities. Implementation of preclinical basic history taking (HT) and physical examination (PE) skills training has been recommended, as it appears to be associated with increased confidence and improved student performance in clerkship. ${ }^{1}$ It has been established that on-campus medical students enjoy the clinical skills programme as it makes them 'feel like doctors'.?

The specialty of internal medicine (IM) historically has been described as 'the mother of all clinical divisions', on which the fundamental instruction of the art of medicine is built. ${ }^{3}$ Thus students' skills in other areas are developed later and differentiated into other clerkships and skill sets. This may account for why IM faculty members are heavily involved in teaching the "primary care clinical skills courses' in primary healthcare centres (PHCCs) and hospitals. ${ }^{4}$ Approximately one-third of faculty members in the colleges are internists. Among the IM primary care abilities, HT and PE are basic skills requiring training in the college programme. Hence a well organised IM orientated preclinical skills curriculum is crucial for enhancement of students' future comfort and confidence with PHCC patients and hospital inpatients during their clinical clerkship. ${ }^{5}$ Nonetheless, recent studies have suggested that the HT and PE skills of clinical clerks is already below expectation at the end of the IM clerkship and residency. ${ }^{6-8}$

Accordingly, this study aims to evaluate the effectiveness of parallel on-campus, faculty led, student assisted training and PHCC basic HT and PE skills training courses on pre-clerks' preparedness for IM clerkship, especially confidence/coping, patient management and self-directed learning. Particularly, the immediate and lasting effects of PHCC training and PHCC training in combination with on-campus HT and PE training modules (PHCC+on-campus) were assessed by group objective structured clinical examination (GOSCE) and objective structured clinical examination (OSCE), as well as self-assessed hospital practice preparedness, to assess the possibility of future application in preclinical skills curriculum.

\section{METHODS}

\section{Study design, setting and participants}

This prospective comparative study was conducted with pre-clerks in academic years 2014 and 2015 at the Faculty of Medicine, Yang-Ming University, Taipei, Taiwan, which is one of the three largest government funded medical schools supervised by the Taiwan Medical College Accreditation Council for curriculum development and quality assurance. In their preclinical years, pre-clerks were recruited on a voluntary basis to receive additional training, GOSCE/OSCE, self-assessments and follow-up until the beginning of clerkship. Preparation for this interventional programme lasted for almost 1 year and was done by a group of IM department members of Taipei Veterans General Hospital (TVGH), which is the teaching hospital of Ying-Ming University. TVGH is one of the largest institutions of clinical medical education in Northern Taiwan and has a National Clinical Skills Training Centre. In addition to examining every aspect of the modules, organisers developed serial specific chief complaint based clinical scenarios to intensify learner's basic IM HT and PE skills. Ethics approval was obtained from the ethics committee of our institution, and care was taken to apply the World Medical Association Declaration of Helsinki principles to the research.

\section{PHCC module (control)}

A total of 64 pre-clerk volunteers (academic year 2014), randomly divided into eight groups ( $\mathrm{n}=8$ in each group), were exposed to a PHCC module, comprising 7 week PHCC weekly sessions and 7 week regular pre-clerkship sessions (figure 1).

\section{PHCC+on-campus module}

In academic year 2015, a PHCC+on-campus basic HT and PE skills training module was developed for 64 pre-clerk volunteers who were randomly divided into eight groups ( $\mathrm{n}=8$ in each group). In parallel, the PHCC+on-campus module was designed as having an on-campus lecture and small group practice in 1 week, followed by a PHCC session the following week (figure 1). In order to establish clinical skills smoothly, head and neck (H-N), cardiovascular (CV), chest and abdomen specific HT and PE on-campus structured training sessions were arranged after they had finished their corresponding blocks (including lecture, critical thinking and differential diagnosis training) (figure 2).

In addition to intensive training for weekly sessions, all faculty members received a comprehensive teaching manual containing general, $\mathrm{H}-\mathrm{N}, \mathrm{CV}$, chest and abdomen focused HT and PE, detailed case scenarios, major/minor learning issues, checklists, lecture notes and videos. Additionally, an introduction to structured and motivated feedback techniques was given in the reference booklets. To ensure standardised teaching/ feedback during on-campus sessions, consensus on how to lead each session was achieved among faculty members with different levels of experience.

Before each weekly on-campus session, the aforementioned teaching/learning materials for faculty members also were mailed to students for self-directed learning before class and for future reference. The on-campus part of the PHCC+on-campus module followed the 'see a good one do a better one' strategy. ${ }^{9}$ During dyad practice, pre-clerks were 


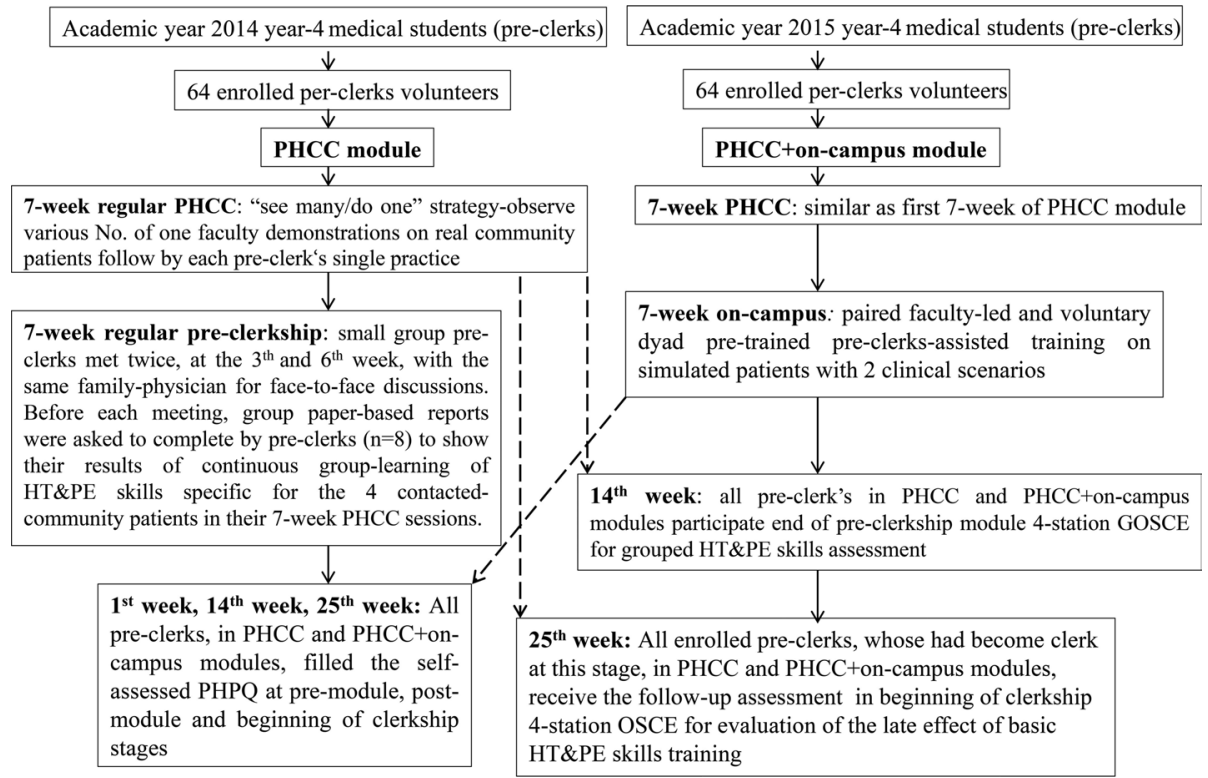

Figure 1 Flowchart for the primary healthcare centre (PHCC) basic history taking $(\mathrm{HT})$ and physical examination (PE) skills training module in academic year 2014. Both weekly PHCC and on-campus sessions were run as small groups $(n=8)$ of preclerks and their specific faculty within 4 hours. At the second 7 week assignment based group learning stage, small group preclerks met twice, at the third and sixth weeks after the 7 week PHCC weekly sessions, with the same family physician for face to face discussions of their continuous group learning of $\mathrm{HT}$ and PE skills specific to the community contacted patients within their first stage (7 week PHCC sessions).GOSCE, group objective structured clinical examination; OSCE, objective structured clinical examination (OSCE).

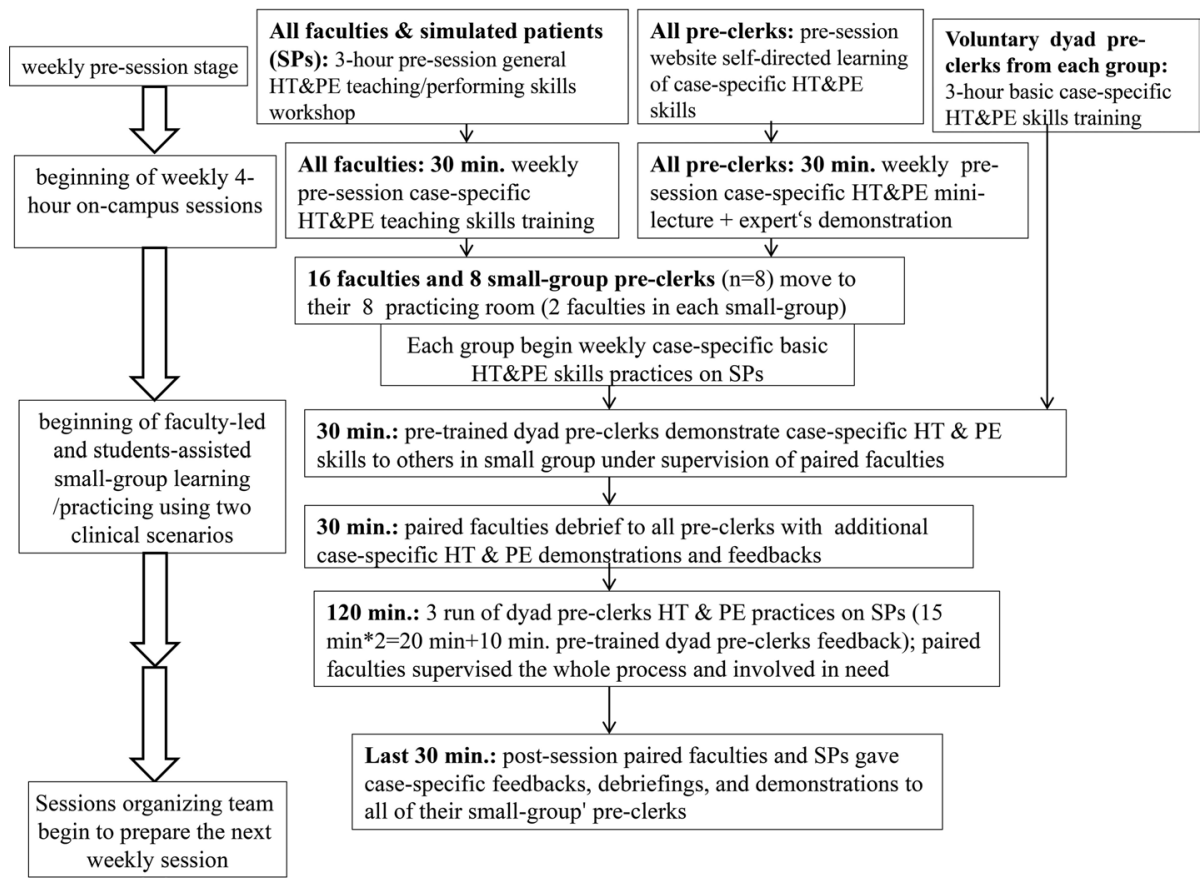

Figure 2 Flow chart for the primary healthcare centre (PHCC) in combination with on-campus basic history taking (HT) and physical examination (PE) skills training module in academic year 2015.

instructed to alternate actively between HT and PE parts, and instructed to observe their partner. The first pre-trained dyad students, who were responsible for HT and PE demonstration, received pre-class tutorials for preparation to provide their peers with precise/ corrected case based HT and PE skills (figure 2).

\section{GOSCE and OSCE raters, SPs and student training}

Raters and simulated patients (SPs) were walked through the forms and the teaching points of each station prior to the end of the pre-clerkship module GOSCE and at the beginning of the clerkship OSCE. All raters, who were blinded to the aim of the study and its design, were 
randomly assigned to GOSCE/OSCE stations. Prior to the GOSCE/OSCE, the enrolled pre-clerks met in a conference room to view the structure and objectives of the GOSCE/OSCE.

\section{End of the pre-clerkship module GOSCE}

The GOSCE was designed as a formative experience where pre-clerks were encouraged to learn from each other by performing IM related HT and PE on SPs. Simultaneously, all enrolled pre-clerks were divided into eight groups ( $\mathrm{n}=8$ in each group) to run eight GOSCE stations, in two parallel tracks (see online supplementary table 1) and to be evaluated with an assessment form (see online supplemental table 2).

\section{Beginning of clerkship OSCE}

After finishing their pre-clerkship, all enrolled pre-clerks commenced their clerkship at TVGH and received OSCE. All enrolled pre-clerks had no further HT and PE skills training between the end of the pre-clerk module and the beginning of their clerkship. Therefore, we used the beginning of their clerkship OSCE to assess the late effects of either PHCC or PHCC+on-campus by comparison of the beginning of clerk OSCE performance of each pre-clerk with their earlier end of pre-clerk module GOSCE performance. Using a specific assessment form (see online supplemental table 3), each clinical clerk needed to run four OSCE stations (H-N, CV, chest and abdomen), as showed in online supplemental table 4 .

\section{Self-reported PHPQ}

The Preparation for Hospital Practice Questionnaire (PHPQ) is a valid and reliable 41 item self-reported questionnaire containing eight subscales (interpersonal skills, confidence/coping, collaboration, patient management and practical skills, understanding science, prevention, holistic care and self-directed learning) designed to assess key areas of medical hospital practice independently in different clinical settings. ${ }^{10}$ In this study, the 17 items assessing capabilities in the confidence/coping, patient management/practical skills and self-directed learning (evaluation of performance, identification of learning needs) subscales of the PHPQ were completed by all enrolled learners (see online supplemental table 5).

All enrolled learners in PHCC and PHCC+on-campus module were asked to finish the same selected items in the PHPQ at the start of the module (week 1), at the end of the module (week 14) and at the beginning of their clerkship (week 25).

\section{Statistical analysis}

The information gathered from different stages was input and analysed using the Statistical Package of Social Sciences (SPSS, V.20.0). Data are presented as mean $\pm \mathrm{SD}$ or medium (IQR). A two sample $t$ test, oneway ANOVA and the Mann-Whitney U test were used to study significant difference between numerical or ordinal data between groups. A p value $<0.05$ was considered statistically significant.

\section{RESULTS}

Table 1 shows that weekly session hours, number of pre-clerks in each small group, time point to complete the GOSCE, OSCE and sequential HHPQ, total enrolled pre-clerks, gender distribution, mean age and percentage of participants with previous HT\&PE trainings were similar between the PHCC and PHCC+on-campus modules.

\section{Group's performance at end of pre-clerkship module GOSCE stations}

In comparison with the PHCC group, the ' $\mathrm{HT}$ and PE communication/care skills, techniques, item content and time control' were significantly improved in the PHCC+on-campus group (table 2). Nonetheless, the PHCC group performed significantly better in the HT and PE sequence compared with the PHCC+oncampus group. In addition to better performance in $\mathrm{H}-\mathrm{N}, \mathrm{CV}$, chest and abdomen GOSCE stations, a significantly higher proportion of grouped pre-clerks had high OSCE scores $(>300)$ in the PHCC+on-campus module than those in the PHCC module (figure $3 \mathrm{~A}$ and table 3). The grouped students' CV GOSCE station performance, however, was significantly lower than the other three GOSCE stations in both the PHCC and PHCC+on-campus modules (table 3). The inter-rater reliability for the GOSCE scores was acceptable $(0.81$, $\mathrm{p}=0.02)$.

\section{Individual pre-clerk's performance at the beginning of clerkship OSCE stations}

The inter-rater reliability for the OSCE scores was 0.87 $(\mathrm{p}=0.03)$. In OSCE, the overall HT and PE performance of PHCC module participants was better than PHCC+on-campus participants. Nonetheless, among PHCC module participants, their average beginning of clerkship OSCE performance on HT and PE 'technique, item content and time control scores' were poorer than their early end of module grouped GOSCE performance (table 2). In addition, the PHCC module participants' 'H-N and abdomen' beginning of clerkship OSCE performance were decreased. Among PHCC+oncampus module participants, however, the average beginning of clerkship OSCE performance on HT and PE 'sequence, item content and time control' was better than the early end of module grouped GOSCE performance. Further, in comparison with their end of module grouped GOSCE performance, the PHCC+oncampus module participants' 'CV, chest and abdomen' beginning of clerkship OSCE performance increased (table 3). Finally, a significantly higher proportion of individuals attained high OSCE scores $(>300)$ in the PHCC+on-campus module than those in the PHCC module (figure $3 \mathrm{~A}$ ). 
Table 1 Comparisons of the PHCC and PHCC+on-campus modules

PHCC module

\section{PHCC+on-campus module}

\begin{tabular}{ll}
\hline Weekly session hours & 4 \\
No of pre-clerks in each group & 8 \\
\hline Cases in each weekly PE session & $\begin{array}{l}\text { Varying number of real patient } \\
\text { encounters in the community }\end{array}$ \\
Weekly session faculties & $\begin{array}{l}\text { One family medicine faculty lead } \\
\text { small group students }\end{array}$
\end{tabular}
4 8

8

No real patient encounters; two clinical scenarios by simulated patients

One family medicine faculty lead Two IM faculty members lead and supervise the small group $(n=8)$ practice with feedback/ debriefing at the beginning and end of the session

$\begin{array}{ll}\begin{array}{l}\text { Ways of weekly basic IM HT and PE skills } \\ \text { practice }\end{array} & \begin{array}{l}\text { Grouped pre-clerks observe } \\ \text { faculty conduct HT and PE skills } \\ \text { followed by single practice }\end{array}\end{array}$

Dyad pre-trained learner's demo and dyad followed by single practice pre-trained pre-clerks feedback between each dyad practice

Time point to receive GOSCE after entering $\quad 14 \quad 14$

module (week)

\begin{tabular}{|c|c|c|}
\hline $\begin{array}{l}\text { Time point to receive OSCE after entering } \\
\text { module (week) }\end{array}$ & 25 & 25 \\
\hline $\begin{array}{l}\text { Time points to complete self-assessed PHPQ } \\
\text { after entering module (week) }\end{array}$ & $1,14,25$ & $1,14,25$ \\
\hline $\begin{array}{l}\text { No of enrolled pre-clerks (\% among all pre- } \\
\text { clerks of academic year) }\end{array}$ & $64(67.8 \%)$ & $64(68.4 \%)$ \\
\hline Sex (men/women) & $45 / 19$ & $44 / 20$ \\
\hline Age (years) & $23.8 \pm 2.9$ & $26.1 \pm 1.3$ \\
\hline $\begin{array}{l}\text { Previous experience* with HT training (yes/ } \\
\text { no) }\end{array}$ & $6 / 58$ & $6 / 58$ \\
\hline
\end{tabular}

no)

Previous experience* with PE training (yes/no) 3/61

$4 / 60$

*Defined as at least three previous formal training sessions for history taking/physical examination skills.

GOSCE, group objective structured clinical examination; HT, history taking; IM, internal medicine; OSCE, objective structured clinical examination; PE, physical examination; PHCC, primary healthcare centre; PHPQ, Preparation for Hospital Practice Questionnaire.

\section{Self-reported hospital practice preparedness}

At the pre-clerkship module stage, the self-assessed preparedness in confidence/coping, patient management (PM) and self-directed learning (SDL) domains were similar between the PHCC and PHCC+on-campus pre-clerks (figure $3 \mathrm{~B}$ ). In contrast with the PHCC module learners, higher post-module PHPQ confidence/coping, $\mathrm{PM}$ and SDL scores than their corresponding pre-module scores were noted in the PHCC+on-campus module pre-clerks. Comparing post-module PHPQ confidence/ coping and PM scores, the trend for improvement in these two scores at the beginning of the clerkship stage was higher among PHCC+on-campus module pre-clerks than the trend in PHCC module pre-clerks (figure 3B-D). In other words, the late positive effect of the PHCC+oncampus module on PHPQ confidence/coping and SLD scores was better than those in PHCC module pre-clerks (figure 3B-D).

Additionally, the comparable positive late training effectiveness of PHCC and PHCC+on-campus modules was noted in the 'PM' domain at the beginning of the clerkship (follow-up) stage (figure 3B-D). Both for PHCC and PHCC+on-campus module pre-clerks, the gradual improvement in PHPQ PM scores was accompanied by a progressive increase in PHPQ confidence/coping and PHPQ SDL scores at this follow-up stage.

\section{Correlation between self-assessed hospital} practice preparedness and rater assessed OSCE performance at the beginning of the clerkship stage

In this correlation analysis, we defined high OSCE pre-clerks as having OSCE scores higher than the mean value of pre-clerks in the same module and defined low OSCE pre-clerks as the opposite. In figure 3E-F, high OSCE pre-clerks had significantly higher mean PHPQ confidence/ coping scores than those in low OSCE pre-clerks in PHCG and PHCC+on-campus modules. Nonetheless, the association between high mean PHPQ SDL scores and high OSCE scores was observed only among PHCC+on-campus module participants. In contrast, the mean PHPQ PM scores were not different between high OSCE and low OSCE pre-clerks in PHCC and PHCC+on-campus modules.

\section{DISCUSSION}

As medical education becomes more streamlined with evolving work hour restrictions, preclinical basic 


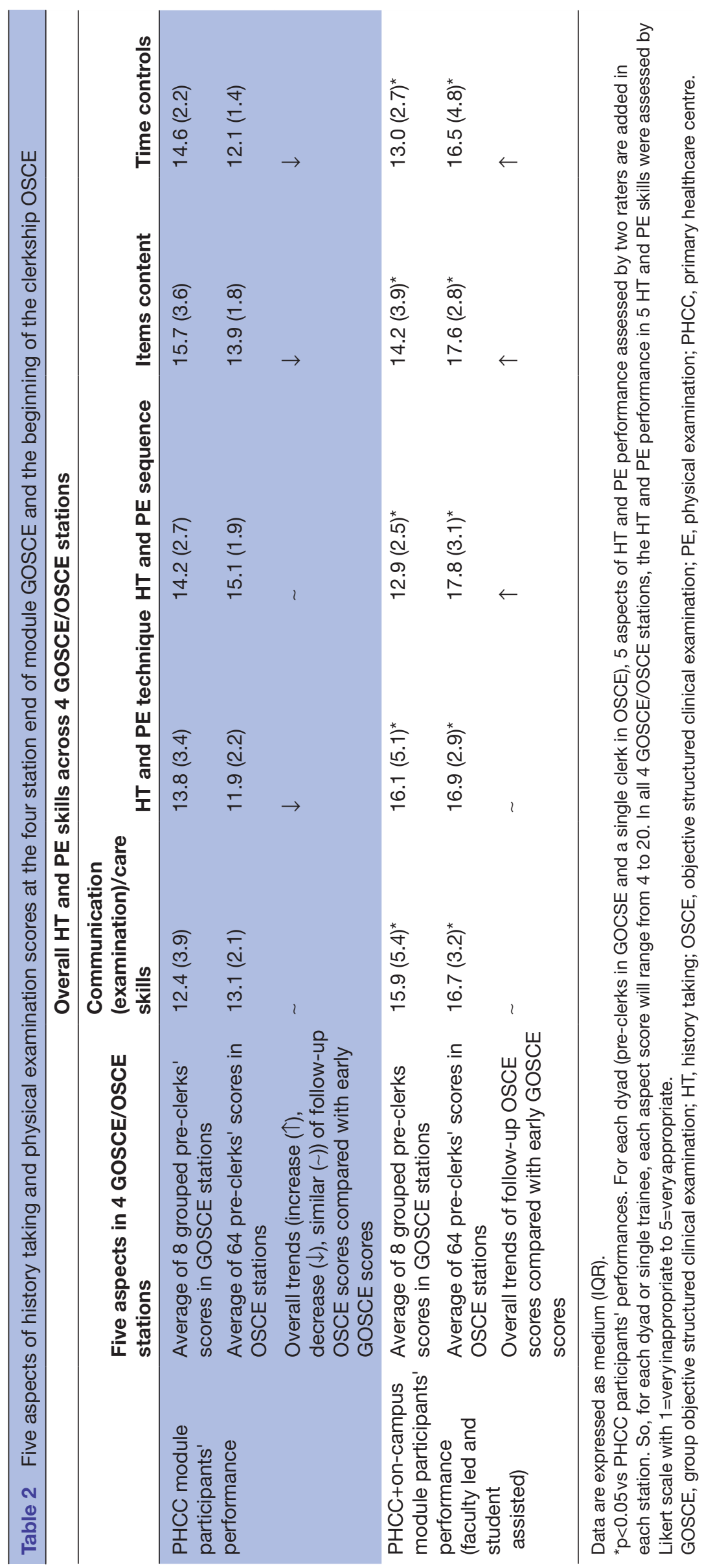




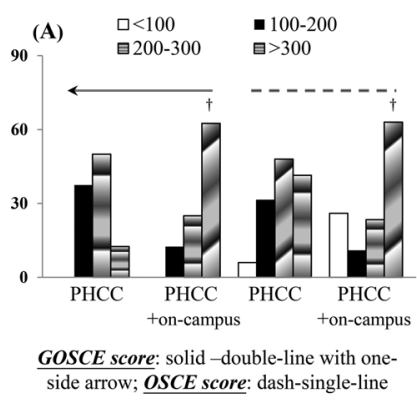

(D). Degree of $\uparrow$ in HPHQ scores

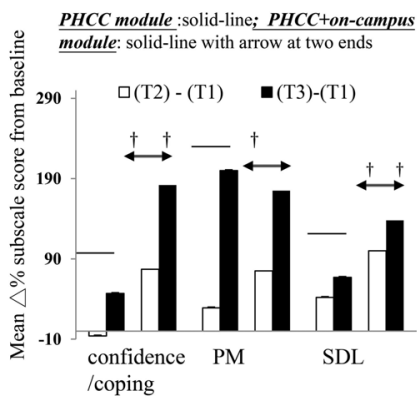

(B). PHCC pre-clerk's PHPQ scores

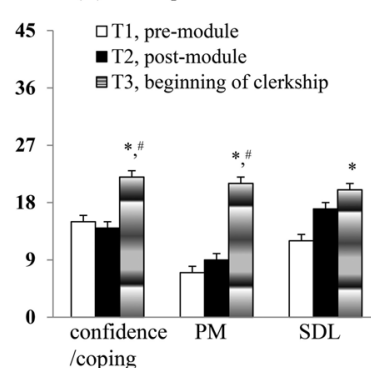

(C). PHCC+on-campus pre-clerk's PHPQ scores

$\square \mathrm{T} 1$, pre-module -T2, post-module

日T3, beginning-of-clerkship

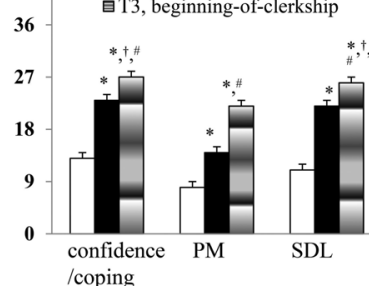

coping

Figure 3 Distribution of (A) grouped pre-clerks' end of pre-clerkship group objective structured clinical examination (GOSCE) and individual pre-clerk beginning of clerkship objective structured clinical examination (OSCE) scores of PHCC and PHCC+oncampus module participants. (B-D). Comparison the impact of primary healthcare centre (PHCC) and PHCC+on-campus history taking $(\mathrm{HT})$ and physical examination (PE) training modules on the sequential changes of the self-assessed Preparation for Hospital Practice Questionnaire (PHPQ) scores for 'confidence/coping,' 'patient management (PM)' and 'self-directed learning (SDL)' subscales. (E-F). Mean PHPQ confidence/coping, PHPQ-PM and PHPQ-SDL scores among high OSCE pre-clerks (beginning of clerkship OSCE scores higher than mean level in the same module) and low OSCE pre-clerks. ${ }^{*} \mathrm{p}<0.05$ vs premodule score; $" p<0.05, p<0.01$ vs post-module score; $\uparrow p<0.05$ vs PHCC module pre-clerks data; $\ddagger p<0.05$ vs low OSCE preclerks.

IM related HT and PE skills training is playing a pivotal role in preparing students for clerkship. For years, considerable feedback from faculty members and students has suggested that most preclinical clerks have difficulty taking a proper history and conducting appropriate physical examinations during their IM clerkship. This study compared the effectiveness of PHCC and PHCC+oncampus modules for augmentation of pre-clerks' IM HT and PE skills, which were evaluated at the end of module GOSCE, at the beginning of the clerkship OSCE and by serial self-assessment of hospital practice preparedness during clerkship. In our study, PHCC+on-campus pre-clerks valued this small group dyad practising approach as instructive and helpful to augment their PHCC learned basic PE skills by identification of points that need to be improved.

Additionally, courses directors reported the decline in basic IM related HT and PE skills over time when these skills are taught early in the curriculum. So, our PHCC and PHCC+on-campus modules were extended over the entire fourth year of medical education. GOSCE provides an opportunity to gain clinical experience by peer observation. GOSCEs also are a resource effective tool for evaluation of basic HT and PE skills as they cost less and require fewer raters per learner than a traditional OSCE. ${ }^{11-13}$ Enrolled pre-clerks in our study reported that end of module GOSCE and follow-up beginning of clerkship OSCE provided them the opportunities for self-reflection and the motivation for continuous bedside learning.

Preclinical students, with limited clinical experience, need intense supervision during the learning process in PHCC, a setting with a variety of patients to practice basic HT and PE skills training. Hence our PHCC+oncampus module is characterised by sufficient on-campus learning resource and continuous community real cases exposure. Notably, our integrated PHCC+on-campus model gradually prepares pre-clerks by linking their PHCC and on-campus learnt skills with inhospital clerkship, which have relatively more ill patients than PHCC. By initial PHCC, on-campus stimulation and activating pre-clerk's continuous SDL, general improvement in HT and PE skills on the follow-up OSCE, as well as on hospital practice preparedness, was found in our study. In particular, additional benefits of on-campus courses are specifically designed sessions and dedication of simulated patients responsible for performing a variety of clinical scenarios. Most of the dedicated faculty members in PHCC+on-campus courses reported that the pre-clerks went through the whole programme with enthusiasm and dedication.

In our weekly HT and PE sessions of PHCC+on-campus module, every pre-clerk had repetitive training for skills within 4 hours. This is because previous studies have 


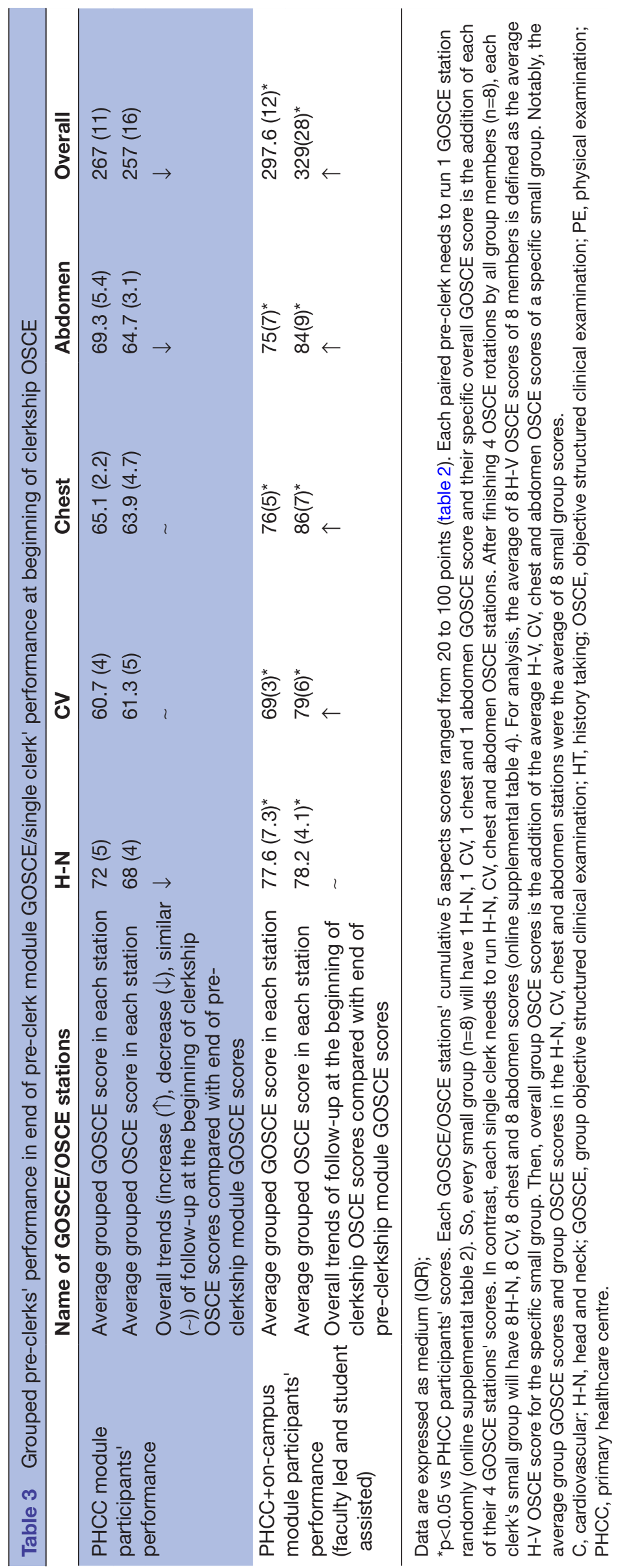


reported that repetitive skills training and expert feedback increase the long term effectiveness of skills training. ${ }^{14} 15$

Medical students need to acquire a number of learning skills, such as confidence and preparation for lifelong SDL. ${ }^{16}$ Within the protected on-campus environment, the well trained structured feedback and debriefing skills by faculty members and SPs increased PHCC+on-campus trainees' PHPQ confidence/coping scores. The active involvement of each pre-clerk in demonstrating clinical scenario based HT and E skills resulted in the late positive effects of the PHCC+on-campus model on pre-clerks' PHPQ-SLD scores. This observation is compatible with the previous report that peer assisted and SDL enhanced the learning efficiency of complex skills in medical education. ${ }^{17}$ In contrast with the rotation of GOSCE station by dyad pre-clerks, every pre-clerk needs to run and complete the task in the four OSCE stations independently. Among participants in the PHCC+on0campus module, the positive association between mean PHPQ SDL scores and high OSCE scores further confirms the role of OSCE to examine the skill performance and SDL ability of each clerk.

It has been reported that observing peers' performances can enhance the confidence and proficiency of learner $\mathrm{HT}$ and $\mathrm{PE}$, which is a mix of cognitive and motor skills. ${ }^{9} 1819$ In our study, PHCC+on-campus module's pre-clerks had opportunities to make repeated observations of their peers' performances on simulated patients. Taken together, it is reasonable to observe the lack of skills degradation over time in OSCE performance and PHPQ SDL scores among the PHCC+on-campus group in our study. In contrast, pre-clerks practising in PHCC are alone due to limited timed per patient encounter. Moreover, training in PHCC has been characterised as opportunistic with an uncontrolled mix of cases, which interferes with the standardisation of training. Compatible with previous observations, our study suggests that the CV HT and PE skills are not easy to improve by PHCC or on-campus training courses alone. ${ }^{6}{ }^{20}$ These results suggest the need for further training of CV PE skills in IM internship and residency.

The PHCC module in our current study was 7 weekly PHCC sessions followed by 7 week assignment based group learning before GOSCE and OSCE. As the positive roles of small group PHCC sessions has been established in training of basic PT and PE skills, ${ }^{51}$ in our study, the PHCC+on-campus module was conducted as 7 week PHCC and 7 week on-campus weekly sessions that ran every 2 weeks alternatively (figure 1 ). It is possible that the length and modules of the PHCC sessions as well as additional resources of PHCC+on-campus module will affect the degree of improvements in the learning outcomes of pre-clerks' HT and PE skills. Therefore, a third group who receive 14 weeks of pure and consistent PHCC training should be included in future studies.

With regard to generalisability, our study design was based on the positive roles of PHCC+skills lab training in Indonesian and Dutch preclinical students. ${ }^{5}{ }^{21}$
Nonetheless, positive results for our PHCC+on campus module are limited to Chinese pre-clerks; the degree to which this parallel module can be extrapolated to training in other countries needs to be validated in a future large scale multicentre study.

\section{CONCLUSIONS}

This prospective comparative study suggests that an integrated PHCC+on-campus module can train preclinical clerk's history taking and physical examination skills effectively and intensify their self-directed learning and patient management abilities to prepare for hospital practice in clerkship.

\section{Author affiliations}

${ }^{1}$ Department of Medicine, National Yang-Ming University, Taipei, Taiwan

${ }^{2}$ Division of Clinical Skills Training, Taipei Veterans General Hospital, Taipei, Taiwan

${ }^{3}$ Division of General Medicine, Department of Medicine, Taipei Veterans General

Hospital, Taipei, Taiwan

${ }^{4}$ Taipei Veterans General Hospital, Taipei, Taiwan

${ }^{5}$ Department of Medical Education, Taipei Veterans General Hospital, Taipei, Taiwan

Acknowledgements The authors express their gratitude to all members of the Faulty of Medicine, Ying-Ming University and Division of Clinical Skills Training, Department of Medical Education, Taipei Veterans General Hospital, Taipei, Taiwan, for their input to this article. The authors gratefully appreciate Professor Ralph Kirby's help in the English writing of the manuscript.

Contributors All authors have read and approved the final manuscript. Y-YY conceived and designed the study, recruited the study subjects, and planned and performed the statistical analysis. S-JW made critical revisions to the manuscript. $\mathrm{L}-\mathrm{YY}$ conceived and designed the study. J-FL performed the analysis and interpretation of the data, and wrote the draft. C-CH recruited the study subjects, and performed the analysis and interpretation of the data. J-FL was responsible for acquisition, analysis and interpretation of the data. F-YL contributed to study supervision and critical revision of the manuscript. S-JH contributed to study supervision. Chin- $\mathrm{CH}$ was responsible for acquisition of the data. RK contributed to critical revision of the manuscript.

Funding This work was supported by the government Ministry of Health and Welfare and Taiwan Association of Medical Education (TAME) and V105EA-005 of TVGH from the Taipei Veterans General Hospital, Taipei, Taiwan.

\section{Competing interests None declared.}

Ethics approval The study was approved by the ethics committee of Taipei Veteran General Hospital.

Provenance and peer review Not commissioned; externally peer reviewed. Data sharing statement № additional data available.

Open Access This is an Open Access article distributed in accordance with the Creative Commons Attribution Non Commercial (CC BY-NC 4.0) license, which permits others to distribute, remix, adapt, build upon this work non-commercially, and license their derivative works on different terms, provided the original work is properly cited and the use is non-commercial. See: http://creativecommons.org/ licenses/by-nc/4.0/

C Article author(s) (or their employer(s) unless otherwise stated in the text of the article) 2017. All rights reserved. No commercial use is permitted unless otherwise expressly granted.

\section{REFERENCES}

1. Jackson MB, Keen M, Wenrich MD, et al. Impact of a pre-clinical clinical skills curriculum on student performance in third-year clerkships. J Gen Intern Med 2009;24:929-33.

2. Lam TP, Irwin M, Chow LW, et al. Early introduction of clinical skills teaching in a medical curriculum--factors affecting students' learning. Med Educ 2002;36:233-40. 
3. Flexner A. Medical education in the United States and Canada. The Carnegie Foundation for the Advancement of Teaching. New York: Madison Avenue; 1910.

4. Omori DM, Wong RY, Antonelli MA, et al. Introduction to clinical medicine: a time for consensus and integration. Am J Med 2005;118:189-94.

5. Widyandana D, Majoor GD, Scherpbier AJ. Effects of partial substitution of pre-clinical skills training by attachments to primary health care centers: an experimental study. Med Teach 2011;33:e313-7.

6. Shapiro J, Rakhra P, Wong A. The stories they tell: How third year medical students portray patients, family members, physicians, and themselves in difficult encounters. Med Teach 2016;38:1033-40.

7. Haring CM, Cools BM, van der Meer JW, et al. Student performance of the general physical examination in internal medicine: an observational study. BMC Med Educ 2014;14:73.

8. Li JT. Assessment of basic physical examination skills of internal medicine residents. Acad Med 1994;69:296-9.

9. St-Onge C, Martineau B, Harvey A, et al. From see one do one, to see a good one do a better one: learning physical examination skills through peer observation. Teach Learn Med 2013;25:195-200.

10. Hill J, Rolfe IE, Pearson SA, et al. Do junior doctors feel they are prepared for hospital practice? A study of graduates from traditional and non-traditional medical schools. Med Educ 1998;32:19-24.

11. Biran LA. Self-assessment and learning through GOSCE (group objective structured clinical examination). Med Educ 1991;25:475-9.

12. Elliot DL, Fields SA, Keenen TL, et al. Use of a with first year medica students. Acad Med 1994;69:990-2.
13. Konopasel L, Kelly KV, Bylund CL, et al. The group: building communication skills in the clinical reasoning context. Patient Educ Couns 2014;96:79-85.

14. Bosse HM, Mohr J, Buss B, et al. The benefit of repetitive skills training and frequency of expert feedback in the early acquisition of procedural skills. BMC Med Educ 2015;15:22.

15. Herrmann-Werner A, Nikendei C, Keifenheim K, et al. "Best practice" skills lab training vs. a "see one, do one" approach in undergraduate medical education: an RCT on students' long-term ability to perform procedural clinical skills. PLoS One 2013;8:e76354.

16. Jacobs JL, Samarasekera DD, Shen L, et al. Encouraging an environment to nurture lifelong learning: an Asian experience. Med Teach 2014;36:164-8.

17. Steele DJ, Medder JD, Turner P. A comparison of learning outcomes and attitudes in student- versus faculty-led problem-based learning: an experimental study. Med Educ 2000;34:23-9.

18. Martineau B, Mamede S, St-Onge $C$, et al. To observe or not to observe peers when learning physical examination skills; that is the question. BMC Med Educ 2013;13:55.

19. Tolsgaard MG, Bjørck S, Rasmussen MB, et al. Improving efficiency of clinical skills training: a randomized trial. J Gen Intern Med 2013;28:1072-7.

20. Williams RG, Klamen DL, Mayer D, et al. A sampling strategy for promoting and assessing medical student retention of physical examination skills. Acad Med 2007;82:S22-5.

21. Widyandana D, Majoor G, Scherpbier A. Preclinical students' experiences in early clerkships after skills training partly offered in primary health care centers: a qualitative study from Indonesia. BMC Med Educ 2012;12:35. 